\title{
Questioni Per la LetTeratura Comparata: uno Sguardo alle Opere di Ignazio Silone e Graciliano Ramos
}

\section{Patricia Peterle ${ }^{1}$}

ABSTRACT: Questo testo ha lo scopo di ripercorrere la traiettoria intellettuale e letteraria di due scrittori, Ignazio Silone e Graciliano Ramos, che hanno vissuto e sperimentato momenti culturali e sociali significativi dei loro paesi di origine: il Brasile dell'Estado Novo e l'Italia del Fascismo. Questo saggio propone, così, uno sguardo che integra e non allontana i profili dell'intellettuale e dello scrittore e che presenta una rilettura del percorso e della produzione letteraria dei due autori.

PAROLE-CHIAVE: Ignazio Silone; Graciliano Ramos; letteratura comparata; intelettuale. 
RESUMO: Este trabalho tem por objetivo percorrer a trajetória intelectual e literária de dois escritores, Ignazio Silone e Graciliano Ramos, que viveram e experienciaram momentos culturais e sociais significativos em seus países de origem: o Brasil do Estado Novo e a Itália do Fascismo. Este ensaio propõe, assim, um olhar que integra e não distancia os perfis do intelectual e do escritor e apresenta uma releitura do percurso e da produção literária desses autores.

PALAVRAS-CHAVE: Ignazio Silone; Graciliano Ramos; literatura comparada; intelectual.

ABSTRACT: This paper traces the intellectual and literary's trajectory of two writers, Ignazio Silone and Graciliano Ramos, each of whom lived and experienced significant social and cultural moments of their countries of origin: Brazil's Estado Novo and Italy's Fascism. This examination paper proposes, in this way, a look that complements and does not remove the profiles of the intellectual and writer, presenting a rereading of the Ignazio Silone's and Graciliano Ramos's footprints and their literary production. KEYWORDS: Ignazio Silone; Graciliano Ramos; comparative literature; intellectual. 


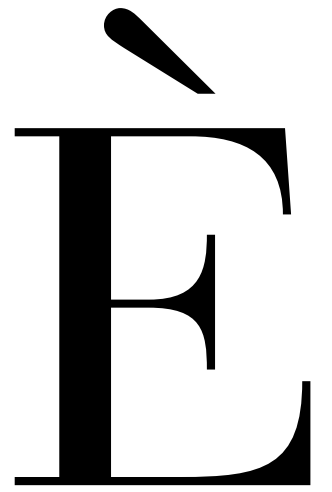

dello scrittore brasiliano la frase: "Não há arte fora da vida, não acredito em romance estratosférico. O escritor está dentro de tudo o que se passa, e se ele está assim, como poderia esquivar-se de influências?"2. Graciliano Ramos ${ }^{3}$ con questa dichiarazione ammette che l'arte e, pertanto, la letteratura, ha una funzione e che questa non è né a margine né disconnessa da tutto quello che la circonda. Cioè: la letteratura non è frutto né è prodotta all'interno di una torre d'avorio; essa può essere anche concepita come il risultato di un intenso legame e rapporto con quello che succede dentro e fuori di casa, per le strade, nel mezzo della folla, negli spazi pubblici e in quelli privati; infine, nelle trame che fanno parte e rappresentano l'esperienza del vissuto.

Resolvo-me a contar, depois de muita hesitação, casos passados há dez anos - e, antes de começar, digo os motivos porque silenciei e porque me decido. Não conservo notas: algumas que tomei foram inutilizadas, e assim, com o decorrer do tempo, ia-me parecendo cada vez mais difícil, quase impossível, redigir esta narrativa. (RAMOS, 1966, p. 33)

2. Intervista col giornalista Ernesto Luiz Maia, pubblicata nella revista Renovação, nel maggio 1944.

3. Libri di Graciliano Ramos tradotti in Itália: Insonnia, 2001, Farenheit451, traduzione di Alessandra Ravetti; Vite Secche, 1993, Biblioteca del Vascello, traduzione di Edoardo Bizzarri - ripubblicato nel 2001 dalla Robin; San Bernardo, 1993, Bollati Boringhieri, traduzione di Oliveira Perlo. 
Queste sono le prime parole con cui Graciliano Ramos inizia la prosa di Memórias do cárcere (1953), una testimonianza che dialoga con gli scritti precedenti, in cui era già possibile rintracciare aspetti memorialistici (come per esempio Infância del 1945, ancora non tradotto in Italia). Quest'opera, ancora inedita in Italia, è il risultato delle vicissitudini dell'autore durante il periodo del regime estadonovista, in cui era stato fatto prigioniero dal 1936 al 1937. Il carcere, con tutte le avversità e difficoltà che può rappresentare una prigione in un regime di dittatura, può essere pure visto come un momento di "isolamento"4 dalla società, fatto questo che offre allo scrittore una riflessione più intensa sul suo essere e sulla sua inserzione nel mondo ${ }^{5}$. Si deve ricordare qui l'ambigua e complessa rete di rapporti che molti intellettuali brasiliani hanno avuto con lo Stato durante i decenni degli anni ' 30 e ' 40.

La celebre espressione di Carlos Drummond de Andrade o "escritor-funcionário" calza per molti artisti, incluso Graciliano Ramos, che, se da un lato hanno bisogno economicamente del lavoro presso lo Stato, dall'altro, rifiutano certe decisioni e atteggiamenti. Nel testo "Infidelidades eletivas: intelectuais e política" (Infedeltà elettive: intellettuali e politica), Helena Bomeny discute il rapporto tra intellettuali e Stato e intellettuali e potere durante il ministero di Gustavo Capanema, dal 1934 al 1945, il quale è riuscito a riunire una serie di personalità quali poeti, scrittori, musicisti, pittori, architetti $^{6}$. Nel caso specifico del Brasile, questo è un momento significativo, proprio perché, se da un lato c’è una forte presenza e attuazione di intellettuali, che più tardi viene denominata e conosciuta come costellazione Capanema, dall'altro è anche il periodo della politica autoritaria del governo. "No Brasil da era Vargas, o dilema da participação dos intelectuais na política teve no ministério de Capanema um de seus momentos memoráveis" (BOMENY, 2001, p. 20).

La rivista Cultura Política, una delle pubblicazioni promosse dall'Estado Novo, legata direttamente al Dipartimento di Stampa e Propaganda (DIP), presentava come primo scopo il divulgare i cambiamenti del paese e le iniziative governamentali. I lavori riguardanti questo periodico prendono corpo nel febbraio del 1941, e, nel marzo dello stesso anno, Graciliano inizia la sua collaborazione scrivendo degli articoli e facendo la revisione dei testi proposti da altri collaboratori.

Come si sa, i contributi dello scrittore brasiliano sono regolari dal 1941 al 1944,

\footnotetext{
4. Le virgolette indicano e raforzzano la contraddizione esistente nel vocabolo isolamento in questo contesto. Se da un lato la prigione isola l'individuo dalla società, dall'altro, l'essere chiuso in un carcere può rappresentare il simbolo massimo dei meccanismi di controllo di una società. Si veda Michel Foucault Microfisica del potere.

5. Vedere Jean-Paul Sartre in Che cos'è la letteratura?

6. Alcuni nomi sono: Carlos Drummond de Andrade, Mario de Andrade, Manuel Bandeira, Lúcio Costa, Oscar Niemeyer, Candido Portinari, Gilberto Freire, Heitor Villa-Lobos, Cecília Meireles, Vinícius de Morais.
} 
anno in cui cessa la pubblicazione di Cultura Política, però in nessun momento egli, anche se fa parte dello staff del sistema, dimostra la tendenza a "celebrare" o seguire organicamente ciò che veniva proposto. Graciliano Ramos ha pubblicato più di 25 testi, che hanno integrato la sezione "Quadros e Costumes do Nordeste", tranne gli ultimi che sono usciti in altre due: "Quadros e Costumes Regionais" e "Quadros Regionais".

Ignazio Silone, al contrario di Graciliano Ramos, ha vissuto intensamente l'esperienza dell'esilio (quasi vent'anni), avendo il suo nome nella lista dei ricercati dalla polizia fascista. È, quindi, da lontano, da una condizione esilica - che può presentarsi pure a quelli che non si spostano e rimangono nella loro terra d'origine -, che l'intellettuale italiano mantiene il contatto con gli amici e compagni di partito, le reminiscenze più care del paese natale. Gli anni dell'esilio svizzero (1928-1944), dopo la militanza nel proprio paese, sono essenziali per il rapporto che man mano affiora tra Secondino Tranquilli/Ignazio Silone e la letteratura. La situazione italiana, a differenza di quella brasiliana, non permette tante ambiguità, una volta che le norme venivano stabilite e regolate dallo stesso regime. Tanti giornali e organi di informazione vengono chiusi dalla polizia fascista, ma alcuni mantengono attività clandestine. In quest'atmosfera non c'è la possibilità del dubbio o dell'ambiguità; il lavoro presso gli organi pubblici richiedeva l'accettare e l'aderire alle idee e immagini propagandate.

Durante l'esilio, Silone mantiene delle rilevanti funzioni dentro al partito, come la sua partecipazione nella commissione italiana inviata alla Terza Internazionale, di cui era membro anche Palmiro Togliatti. Fino alla rottura con il partito, nel 1931, il rapporto tra Silone e la letteratura è mediato dagli scritti specifici per il partito. È solo dopo 1 '“espulsione", testimoniata in alcune lettere e anni più tardi in Uscita di sicurezza (1965), che lo scrittore abruzzese, lontano da tutto quello che finora era stato e aveva rappresentato il partito - "Il partito diventò famiglia scuola chiesa e caserma; all'infuori di esso il mondo restante era tutto da distruggere [...]" (Silone, 2001, 77) -, si avvicina al testo prettamente letterario e vede in esso un modo per continuare a dialogare con se stesso e con il mondo esterno. La narrativa, dunque, diventa, in questo contesto, materia e modo per l'autoriflessione e per la creazione di un canale di comunicazione. 
Parlare di Silone significa anche riprendere la polemica degli ultimi anni ${ }^{7}$, in cui è stato accusato di essere stato una spia della polizia fascista OVRA. Una polemica che ha provocato una serie di discussioni e pubblicazioni ${ }^{8}$ sui giornali e su libri, ma che, per l'assenza di prove, non si regge in piedi. Tuttavia, si deve ammettere che Ignazio Silone ha avuto dei contatti con alcuni membri della polizia politica fascista a causa della situazione del fratello Romolo, arrestato nel carcere di Regina Coeli. Però, non esiste nessun registro che dimostri la tesi dello spionaggio o che egli abbia fornito delle informazioni importanti e cruciali.

In questo modo, ripercorrendo e rileggendo i passi di Graciliano Ramos e Ignazio Silone, è possibile identificare alcuni punti di contatto tra la produzione letteraria di questi autori, così come anche gli atteggiamenti da loro assunti, nonostante il percorso di vita sia stato diverso. La partecipazione ai partiti comunisti dei loro paesi, la produzione di testi con caratteri di denuncia e di protesta contro un ordine sociale - che si presentava come una normalità, ma era di "eccezione" - sono tutti aspetti che avvicinano le attività e le traiettorie di questi intellettuali, anche se si prendono in considerazione un contesto e culture, la brasiliana e l'italiana, che sono diverse e possiedono caratteristiche proprie.

I decenni degli anni ' 30 e ' 40 sono segnati, tanto in America Latina quanto in Europa, da una serie di regimi totalitari, che per i loro tratti e la loro natura fanno sì che $\mathrm{i}$ cittadini e, in particolare, gli intellettuali siano quasi costretti a prendere una posizione per quello che concerne le loro azioni: o sono a favore o sono contro. È in questo momento, in cui la "neutralità" è praticamente impossibile, pur considerando la specifica realtà brasiliana, che Graciliano Ramos e Ignazio Silone producono opere letterarie, testi per la stampa, e hanno, ognuno a modo suo, la possibilità di dialogo con altri intellettuali dentro e fuori del loro paese. Nella traiettoria di vita e nell'attività di questi due intellettuali, è possibile vedere che in alcuni momenti la sfera del pubblico e quella del privato si confondono in una unica e molteplice. Da questo punto di vista, allora, l'inserimento nella società non può essere solo apparente: l'intellettuale deve, con più o meno chiarezza, sapersi muovere. Per Gramsci, in realtà, è questo "agire" a segnare definitivamente la categoria dell'intellettuale, la sua funzione, differenziandola dagli altri individui. Da queste parole, qualsiasi individuo è un intellettuale, perché possie-

\footnotetext{
7. I testi sono stati pubblicati in varie riviste accademiche: Dario Biocca, Ignazio Silone e la Polizia politica. Storia di un "informatore", in Nuova Storia Contemporanea, 3, 1998, p.67-93 e "Tranquilli (nell'ombra)". Ignazio Silone in Francia, in Nuova Storia Contemporanea, 3,1999, p.53-76; de Mauro Canali Il fiduciario Silvestri. Ignazio Silone, i comunisti e la Polizia politica, in Nuova Storia Contemporanea, 1,1999, p.61-86.

8. Come afferma Vittoriano Esposito, Silone é un caso italiano. Dopo il lungo silenzio, il nome dello scrittore abruzzese riappare, alla fine degli anni '90, sui titoli dei supplementi culturali dei giornali italiani di grande circolazione, data 1'accusa di spia della polizia fascista (OVRA) infiltrato nel Partito Comunista. Molti intellettuali hanno negato questa ipotesi: Norberto Bobbio, Indro Montanelli e
} 
de la capacità dell'intelletto, tratto distintivo degli esseri umani rispetto agli animali. Tuttavia, ciò non basta. Godere di questa qualità innata non è, infatti, sufficiente per esercitare il ruolo di intellettuale; ciò che si può dire è che tutti possono essere considerati dei potenziali intellettuali. L'esercizio di questa funzione è, per tutto ciò che è stato detto, intrinsecamente collegato al posto occupato e anche alle attività svolte dal soggetto intellettuale nella società in cui è inserito.

In questa prospettiva, alcune domande che si pongono sono: È possibile tracciare il profilo di questi scrittori come intellettuali? Com'è il rapporto di Silone e Ramos con la società? Qual è il loro rapporto con lo Stato e come sono le loro prese di posizioni davanti ad esso? Come viene rappresentata la società nella loro produzione letteraria? Quali sono le strategie scelte per narrare quello che si vede e viene "testimoniato"? Questo narrare delle esperienze vissute, che certamente passano per un filtro che è quello della memoria e del registro letterario, in questi due casi specifici, può essere visto come un movimento continuo che sottolinea la dualità e la contrapposizione ivi inerente tra "l'immagine sociale e l'immagine intima di se stesso" (ARTIÈRES, 1988, p. 35) - il discorso degli spazi pubblici e privati. È, in effetti, questa problematica che viene fuori quando si pensa alla categoria dell'intellettuale e, ancor di più, allo scrittore-intellettuale che esplora il suo intimo più profondo nelle realizzazioni dei suoi testi e, dopo, li socializza mediante la pubblicazione e la commercializzazione dell'opera. Un'analisi più attenta delle biografie di Silone e Ramos è essenziale per la problematizzazione e interpretazione di tematiche latenti nelle loro opere e progetti letterari.

Puntare l'attenzione sulla produzione letteraria e saggistica di Silone e Ramos, ignorando una cartografia più ampia delle loro inserzioni nelle rispettive società, significa avere uno sguardo parziale, frammentario ed incompleto. È, dunque, vedere una parte e non il tutto come un insieme, che richiede una determinata distanza, la quale permette di identificare e riconoscere, come afferma Pierre Bourdieu, le collocazioni e gli spostamenti nello spazio sociale. I progetti letterari di Silone e Ramos, data la natura dei temi scelti - personaggi e scenari marginali, la miseria, lo sfruttamento, l'abuso di potere...-, non possono essere visti isolatamente. Il rapporto che si stabilisce con il periodo in cui sono stati prodotti e con le problematiche delle rispettive società formano una trama fitta di nodi e tensioni, i quali si stabiliscono tra l'individuo e la società

studiosi come Vittoriano Esposito, Sergio Soave e Giuseppe Tamburrano. Alcuni libri che sono frutto di questo dibattito sono: BIOCCA, D.; CANALI, M.. L'informatore: Silone, i comunisti e la polizia. Diegaro di Cesena: Luni, 2000; BIOCCA, D. Silone. La doppia vita di un italiano. Milano: Rizzoli, 2005; TAMBURRANO, G.. Il “caso Silone”. Torino: UTET, 2006; TAMBURRANO, G. Processo a Silone. La disavventura di un povero cristiano. Bari: Lacaita, 2001. 
nello spazio privato e pubblico e profilano lo spazio che questi autori occupano nel sistema sociale e culturale.

Uscita di sicurezza (1965) di Silone, pubblicato ad una certa distanza di anni dalla fine del regime di Mussolini, rappresenta una specie di "storiografia" del proprio percorso come militante politico e scrittore. In questo libro, Silone racconta/narra la sua versione dei momenti più difficili: il terremoto del ' 15 e la perdita del nucleo famigliare; l'ingresso nella politica; l'inizio del suo rapporto col partito comunista, la partecipazione alla Terza Internazionale, l'inizio dell'allontanamento e l'espulsione dal partito, l'isolamento e la scoperta della scrittura letteraria. Negli anni ' 60 , anche se non era riconosciuto dalla critica italiana, Silone è già un autore rinomato "dentro" e fuori l'Italia. Seguendo questa strada delle memorie, però con alcune differenze, le Memórias do cárcere di Graciliano Ramos non godono della distanza temporale come quelle siloniane, e ciò ha un influsso diretto sull'atto dello scrivere, che si presenta più ordito. Tale testo è idealizzato e scritto in un momento di grande tensione: nel momento stesso in cui vuole denunciare la brutale esperienza del carcere, Graciliano scrive sotto il governo di Getúlio Vargas. Il carcere delle memorie è anche il posto della disumanizzazione, in cui il corpo, fisico e mentale, è proprio il simbolo più emblematico perché, maltrattato e dilacerato, subisce segni visibili ed invisibili indimenticabili. È, senza dubbio, uno spazio in cui le forze occulte ed esistenti del potere si concretizzano. Il corpo, dunque, rappresenta la tensione perché in esso s'iscrivono la repressione, le torture fisiche e mentali, ma anche la resistenza, la denuncia tacita delle violenze subite. In ogni modo, questi due testi biografici, che hanno il tono della testimonianza, possono essere considerati come una rilettura, fatta dagli stessi scrittori attraverso il filtro della memoria, delle vicissitudini esplorate e vissute.

L'immagine della prigione/carcere è un elemento che ripercorre ambedue le opere già citate e ancora altri due romanzi: Fontamara (1933) Vite secche (1936). Quest'opera è la storia di una famiglia del sertão nordestino, una delle zone più povere $\mathrm{e}$ dimenticate del territorio brasiliano, composta dal padre, Fabiano, dalla madre, sinha Vitoria, e dai due figli, che non hanno un nome, detti figlio più grande e figlio più piccolo, che è sempre in movimento. "Trasloco" e "Fuga" sono esattamente il primo e l'ultimo capitolo, che danno già l'idea di un moto continuo. 
Un altro aspetto da evidenziare è il fatto che i due bambini non hanno un nome. Cosa significa avere un nome? Un'identità? Un'esistenza? Si può mettere accanto all'assenza di questi nomi tante altre assenze: assenza di una casa fissa, di educazione, di cibo, di proprietà di linguaggio, di una coscienza della loro stessa esistenza. In linea di massima, questa famiglia può essere il simbolo di un territorio improduttivo, dimenticato, sperduto così come la sua gente. La famiglia è composta anche da un altro membro, Baleia, nome un po' ironico per un cagnolino maltrattato e magro. Tuttavia, questo è un personaggio centrale che si oppone al capo famiglia Fabiano; è lei, la cagnolina, che procura il cibo e caccia le rare cose mangiate dalla famiglia - la fame è quasi una condizione di normalità -, che capisce perfino le sottigliezze e i sentimenti di ognuno di loro. È come se le caratteristiche umane appartenenti a loro quattro, Fabiano, la moglie e i figli, venissero annullate, visto che devono vivere in una specie di selva della siccità a partire dagli istinti, e passassero a Balena, che in tal modo si antropomorfizza.

È, quindi, in questo quadro desolato che Graciliano Ramos inserisce i suoi secchi e duri personaggi che sono sfruttati dai cosiddetti fazendeiros, proprietari delle grandi proprietà agricole, e da tanti altri. L'episodio del carcere è un esempio di questo sfruttamento. Fabiano é portato in carcere senza saperne il motivo e senza capire molto della situazione in cui si trovava. "Se não fosse aquilo... Nem sabia. O fio da ideia cresceu, engrossou - e partiu-se. Difícil pensar" (RAMOS, 2002, p. 36). È con questo stile che Graciliano mette in scena un momento drammatico della narrativa; qui, veramente, Fabiano, che non parla ma grugnisce, che vive "tão agarrado aos bichos", in una situazione di esplorazione e abuso di potere, pur provando che non c'è modo di lottare, ha le idee che non si rafforzano, si spezzano e si rompono. Questo esempio rafforza l'idea di un essere che, anche "avendo coscienza" dei suoi diritti, accetta quello che gli capita senza interrogarsi molto. Un altro episodio è quello in cui Fabiano riceve lo stipendio. Gli vengono addebitate cose non dovute e lui, nella sua limitatezza e semplicità, non può fare altro che accettare. A Fabiano, dunque, non viene data la possibilità di cambiamento, le sue disavventure passeranno ai figli e così via; è qualcosa di ciclico che si ripete di generazione in generazione, come le stagioni dell'anno. In un territorio dominato dalla siccità e dal sole, rappresentato anche nei disegni di Aldemir Martins 
e da alcune copertine, il periodo dell'inverno è sempre l'attesa di una speranza. Però, in questa realtà desertica e quasi micidiale, l'acqua non arriva in maniera auspicata; cioè, tutte le aspettative per l'inverno - l'acqua che avrebbe fatto ricrescere i campi e rivivere gli animali - diventano una delusione, un incubo perché essa arriva violentemente e distrugge le piantagioni e uccide gli animali annegandoli. Quindi, la speranza diventa anche una tragedia. L'immagine finale del libro, nel capitolo intitolato "Fuga", della famiglia di Fabiano che cammina in cerca di un nuovo posto di lavoro, recupera quella iniziale di "Mudança". Tutto ciò riafferma la geometria circolare del romanzo.

A Fontamara, paese immaginario e sperduto nella Marsica, vive una comunità di cafoni, contadini che vivono di quello che gli fornisce la terra, seguendo le ore solari. Una comunità lontana dai centri un po' più grandi, in cui le abitudini passano di generazione in generazione. Questa lontananza non è solo fisica o geografica, è anche culturale; in effetti, la lingua parlata in città non era molto ben capita dai fontamaresi. Come i personaggi di Graciliano Ramos, quelli di Silone presentano un ventaglio di assenze, confermato fin dalla prima riga del romanzo: assenza della luce, dell'acqua, di cibo, d'istruzione. In cerca di lavoro, uno dei narratori insieme a Berardo parte per la grande metropoli romana. Una volta lì, cercano di superare le aspre condizioni in cui si vengono a trovare, finché sono fatti prigionieri dalle milizie fasciste senza capirne il motivo. Ed è in questo momento di totale isolamento che Berardo, personaggio di Silone, e Fabiano, personaggio di Graciliano Ramos, provano un'esperienza molto simile: quella del carcere.

In ambedue le narrative, Fabiano e Berardo hanno di fronte a loro la fragile opportunità di un probabile processo di presa di coscienza di quello che accadeva attorno a loro. Fabiano, come è stato già citato, mentre è da solo in cella, gli vengono in mente alcuni flash, dei dubbi, ma questa fragile possibilità di presa di coscienza si disfa e gli scappa. Egli ritorna alla situazione iniziale, nonostante le domande che lui stesso si pone. Berardo, pur essendo "esiliato" come il primo personaggio di Graciliano Ramos ha come compagno di cella un personaggio che sarà fondamentale per i cambiamenti di tutta la narrativa. Anche se il personaggio siloniano ha la possibilità di crescere, vedere che il mondo non si limita al suo piccolo villaggio e perfino morire in beneficio degli altri, la fine dei fontamaresi è tragica. Il paese viene devastato e distrutto senza 
nessun tipo di pietà verso gli abitanti di quel territorio. I pochi che riescono a scappare dal massacro fuggono, ma poi sono impossibilitati a tornare in una terra che non esiste più. L'ultima frase di questo romanzo, in qualche modo, riprende l'interrogativo e il sentimento di inquietudine che sono anche messi tacitamente accanto ai passi di Fabiano e della sua famiglia da Graciliano Ramos. "Dopo tante pene e tanti lutti, tante lacrime e tante piaghe, tanto odio, tante ingiustizie e tanta disperazione, che fare?" (SILONE, 1985, p.259) È un problema inerente sia alla siccità del sertão nordestino sia alle terre dei cafoni abruzzesi, in un quadro più grande dello sfruttamento dell'essere umano.

Graciliano Ramos e Ignazio Silone hanno come punto di partenza le loro realtà, il nordeste e l'Abruzzo contadino, ma non si limitano ad esse proprio perché le loro tematiche trascendono, senza tralasciare i punti di partenza, i limiti geografici; la loro materia è l'uomo e la sua essenza. Infatti, lo scrittore di Pescina, nella prefazione a Fontamara, firmata Davos 1930, afferma che i lavoratori di tutto il mondo si assomigliano e si capiscono perché parlano la stessa lingua: i felas, coolies, peones e mujiques condividono le stesse condizioni di vita.

Dall'esperienza di questi due autori, quello che si vede è un complesso sistema, formato da innumerevoli rapporti e tensioni. Lo studio della letteratura mondiale/universale $^{9}$ (Letteratura Comparata) dovrebbe puntare anche sulla ricognizione di questi intrecci, che formano dei nodi e intessono questa grande trama che sono le letterature. Un vero macrosistema costituito da tanti altri sistemi più piccoli, ma che mantengono un intenso dialogo concretizzantesi sia direttamente che indirettamente. D'altro canto, non è possibile pensare ad una letteratura che sia solo nazionale, che non presenti nessun collegamento con altri sistemi letterati e culturali, mediante l'intercambio culturale, la lettura, la traduzione o altri mezzi. Il polisistema culturale e letterario si delinea, nel caso di Silone e Ramos, a partire dalle tematiche affrontate, per esempio, anche se poi ricevono dei trattamenti diversi e sono lontane geograficamente.

[...] probably all systems known to us have emerged and developed with interference playing a prominent role [...] there is no tone single literature which did not emerge through interference with a more established literature: and no literature could manage without interference a tone time or another during its history. (EVEN-ZOHAR, 1990, p.59).

9. Vedere le definizioni di Franco Moretti. 
Come mette in risalto Itamar Even-Zohar, il polisistema è composto da vari flussi che tracciano la mobilità, la flessibilità d'informazione. Identificare e cartografare questi flussi non è un compito facile, visto che molte volte sono lì presenti, ma sono invisibili ad una prima lettura e analisi. È forse per questo che Franco Moretti afferma: "Distant reading: where distance, let me repeat it, is a condition of knowledge: it allows you to focus on units that are much smaller or much larger than the text: devices, themes, tropes-or genres and systems" (MORETTI, 2000, p. 57). Certamente, una proposta di analisi di questo tipo può tralasciare alcuni elementi importanti concernenti lo studio letterario. L'ideale, comunque, in questa prospettiva è cercare di trovare l'equilibrio, mantenendo la distanza necessaria per fare uno studio che porti all'interpretazione della testimonianza letteraria di Ignazio Silone e Graciliano Ramos.

Questo tipo di approccio che avvicina contesti culturali, Italia e Brasile, offre la possibilità di vedere nuove prospettive interpretative nel caso specifico di ogni autore e, inoltre, di scoprire nuovi sentieri nell'ambito degli studi della Letteratura Comparata.

\section{Riferimenti bibliografici}

ARTIÈRES, P. Arquivar a própria vida. In Estudos Históricos - Arquivos pessoais, v.1, n.21, 1988.

BOMENY, H. Infidelidade eletivas: intelectuais e política. In Capanema: intelectuais e políticas. São Paulo: Editora FGV, 2001. (org.). Constelação BOURDIER, P. Lições da aula. São Paulo: Editora Ática, 2001.

. A ilusão biográfica. In: FERREIRA, Marieta de Moraes; Amado, Janaína. Usos e abusos da história oral. São Paulo: FGV, 2006.

EVEN-ZOHAR, I. Polysystem Studies. In Poetics Today, n. 11:1, 1990.

FOUCAULT, M. Microfisica del potere. Torino: Einaudi, 1977.

MORETTI, F. Conjectures of World Literature. In New Left Review, n. 1 jane-feb, 2000, p. 57.

RAMOS, G. Vidas Secas. Rio de Janeiro: Record, 2002, p. 6. . Memórias do Cárcere. Vol. I e II. Rio de Janeiro: Record, 1966.

SARTRE, J. Che cos'è la letteratura ed altri scritti. Milano: Il Saggiatore, 2004.

SILONE, I. Fontamara. Milão: Mondadori, 1985, p.259. 